\title{
Lipid Replacement Therapy with a Glycophospholipid Formulation with NADH and CoQ10 Significantly Reduces Fatigue in Intractable Chronic Fatiguing Illnesses and Chronic Lyme Disease Patients
}

\author{
Garth L. Nicolson ${ }^{1 *}$, Robert Settineri ${ }^{2}$, Rita Ellithorpe ${ }^{3}$ \\ ${ }^{1}$ Department of Molecular Pathology, The Institute for Molecular Medicine, Huntington Beach, USA; ${ }^{2}$ Sierra Research, Irvine, USA; \\ ${ }^{3}$ Tustin Longevity Center, Tustin, USA. \\ Email: "gnicolson@immed.org
}

Received January $17^{\text {th }}, 2012$; revised February $25^{\text {th }}$, 2012; accepted March $19^{\text {th }}, 2012$

\begin{abstract}
Objectives: A preliminary open label study was initiated to determine if a combination oral supplement containing a mixture of phosphoglycolipids, coenzyme Q10 and microencapsulated NADH could affect fatigue levels in long-term patients with intractable fatigue. Fatigue was determined by the validated Piper Fatigue Scale before, during and after the trial. Participants included 58 patients (30 females and 28 males) with chronic fatigue syndrome/myalgic encephalomyelitis, chronic Lyme disease or other fatiguing illnesses, such as fibromyalgia syndrome or Gulf War illness. These patients had been symptomatic for an average of $17.1 \pm 0.6$ years, had been seen by many physicians (15.2 \pm 0.7$)$ and had used many other supplements and drugs $(35.4 \pm 2.7)$ without apparent reductions in their fatigue. Results: Participants in the study responded to the combination test supplement, showing a 30.7\% reduction in overall fatigue within 60 days $(P<0.001)$. Analysis of subcategories of fatigue indicated that there were significant improvements in the ability to complete tasks and activities as well as significant improvements in mood and cognitive abilities. Regression analysis of the data indicated that reductions in fatigue were gradual, consistent, and occurred with a high degree of confidence $\left(\mathrm{R}^{2}=0.960\right)$. The data also suggested that further reductions were likely if the participants had continued the supplement beyond the 8-week trial. Males responded slightly better to the combination supplement than females, and the patients with the most severe forms of fatigue responded slightly better than those with milder fatigue, independent of their diagnosis. Conclusions: The combination supplement was a safe and effective method to significantly reduce fatigue in long-term patients with intractable chronic fatigue.
\end{abstract}

Keywords: Fatigue; Chronic Fatigue Syndrome; Lyme Disease; CoQ10; NADH; Phospholipids

\section{Introduction}

In the last few years natural supplements have been used to reduce fatigue in patients with chronic fatigue and other chronic illnesses [1,2]; however, few if any of these natural supplements were considered effective [2]. Chronic or intractable fatigue that is not reversed by sleep is the most common complaint of patients seeking general medical care [3,4]. Fatigue occurs naturally during aging, and it is also an important secondary condition in many chronic diseases [4].

Fatigue and especially chronic fatigue have been described as a multidimensional sensation, and clinical studies have determined the extent of fatigue in aging and in various medical conditions (neurological, respiratory,

\footnotetext{
${ }^{*}$ Corresponding author.
}

coronary, musculoskeletal, metabolic, autoimmune and gastrointestinal diseases, cancer, infections) [3,5-7]. Most patients consider fatigue to be a loss of overall energy and inability to perform even simple tasks without exertion $[3,4]$. At the cellular level fatigue is related to cellular energy systems found primarily in the cellular mitochondria and specifically in the inner mitochondrial membrane electron transport chain $[8,9]$. Damage to mitochondrial components, especially mitochondrial membranes, occurs in various diseases, mainly by oxidation, and this can result in increased ion leakage across mitochondrial membranes and impairment in the ability of mitochondria to produce high-energy molecules needed for survival and growth $[10,11]$.

During aging and most chronic diseases the production of oxidative molecules, such as Reactive Oxygen and 
Nitrogen species (ROS/RNS) that are free radical oxygen- and nitrogen-containing molecules, such as nitric oxide, oxygen and hydroxide radicals and other molecules, can cause oxidative stress and cellular damage [10-12]. This results in oxidation of lipids, proteins and DNA [10-13]. When oxidized, these molecules are structurally and sometimes functionally changed [13,14]. Important targets of ROS/RNS damage are cellular membranes, and in particular, mitochondrial and membranes, especially their phospholipid components [11-14].

In fatiguing illnesses, such as chronic fatigue syndrome/ myalgic encephalomyelitis (CFS/ME), patients have intractable fatigue for at least six months and show increased susceptibility to oxidative stress and peroxidation of membrane lipids $[15,16]$. To some degree this excess oxidative stress can be reduced with antioxidant supplements $[18,19]$, but these antioxidants cannot repair the oxidative damage to cells.

Recent clinical trials have shown the effectiveness of lipid replacement therapy (LRT) plus antioxidants in the treatment of certain clinical disorders and conditions, such as chronic fatigue $[6,12]$. LRT results in the actual replacement of damaged cellular lipids with undamaged (unoxidized) lipids to ensure proper function of cellular structures, mainly cellular and organelle membranes. LRT can result in the cellular delivery of unoxidized, undamaged membrane glycophospholipids in order to replace damaged lipids and restore function to oxidized cellular membranes. Combined with antioxidant supplements, LTR has proven to be an effective method to prevent ROS/ RNS-associated changes in cellular activities and functions and for use in the treatment of various clinical conditions $[6,12,20]$.

Here we tested the results of using a formulation of glycophospholipids, CoQ10 plus microencapsulated NADH and other nutrients on suppression of fatigue during an open label two-month trial. The subjects in this study were long-term chronic illness patients with intractable fatigue that had tried unsuccessfully many drugs and supplements to reduce their fatigue.

\section{Materials and Methods}

\subsection{Materials and Methods}

An open label, institutionally approved, clinical trial was initiated to study the effects of an all-natural supplement on fatigue. The supplement product, ATP Fuel ${ }^{\circledR}$, containing NT Factor $^{\circledR}$, microencapsulated NADH, CoQ10, pro- and pre-biotics and other nutrients (Researched $\mathrm{Nu}$ tritionals, Inc., Los Olivos, CA), is a patent-pending proprietary nutrient complex containing an exogenous source of polyunsaturated phosphatidylcholine, phosphatidyglyerol, phosphatidylserine, phosphatidylinositol, and other membrane phospholipids, as well as coenzyme Q10 (CoQ10), microencapsulated reduced nicotinamide adenine dinucleotide (NADH) and other micronutrients (Table 1). The participants took the suggested daily dose (5 capsules in the morning and 5 at night) for 8 weeks.

Table 1. Test supplement (ATP Fuel ${ }^{\circledR}$ with NTFactor $^{\circledR}$, NADH and CoQ10) ${ }^{\ddagger}$.

\begin{tabular}{lcc}
\hline \multicolumn{1}{c}{ Component } & Amount per Serving & \% Daily Value \\
\hline Vitamin E (as D- $\alpha$-tocopheryl succinate, mixed tocopherols) & $20 \mathrm{IU}$ & 67 \\
NADH and Coenzyme Q10 & $35 \mathrm{mg}$ & $* *$ \\
$\begin{array}{l}\text { Calcium (as dicalcium phosphate, carbonate, pyruvate } \\
\text { borogluconate, ascorbate and dicalcium pantothenate) } \\
\text { phosphorus (as dicalcium phosphate), magnesium } \\
\text { (as magnesium oxide) }\end{array}$ & $260 \mathrm{mg}$ & 13 \\
$\begin{array}{l}\text { Alpha keto glutaric acid, L-tyrosine } \\
\text { L-Carnipure }\end{array}$ & $180 \mathrm{mg}$ (L-carnitine L-tartrate), pantethine & $160 \mathrm{mg}$ \\
NT Factor $^{\circledR \#}$ & $2000 \mathrm{mg}$ & $* *$ \\
\hline
\end{tabular}

${ }^{\ddagger}$ ATP Fuel ${ }^{\circledR}$ is a registered trademark of Researched Nutritionals, Inc., Los Olivos, CA; "Daily values are based on a 2000 calorie per day diet; ${ }^{* *}$ Daily values not established; ${ }^{*}$ NT Factor ${ }^{\mathbb{B}}$ is a nutrient complex extracted from soy lipids and purified using proprietary processes. It is composed only of food and food components: phosphoglycolipids (polyunsaturated phosphatidylcholine, glycolipids and other polyunsaturated phosphatidyl nutrients), Bifido and Lactobaccillus bacteria (freeze-dried and microencapsulated in viable form), growth media (bacterial growth factors and food, including rice bran extract, arginine, beet root fiber, black strap molasses, glycine, magnesium sulfate, para-amino benzoate, leek, pantethine, taurine, garlic, calcium borogluconate, potassium citrate, spriulina, bromelain, natural vitamin E, calcium ascorbate, -lipoic acid, oligosaccharides, vitamin B-6, niacinamide, riboflavin, vitamin B-12, folic acid, inositol, calcium pantotenate, chromium ficolinate). NT Factor ${ }^{\circledR}$ is a registered trademark of Nutritional Therapeutics Inc., Commack, NY. 
Fatigue was scored using the Piper Fatigue Scale (PFS). The PFS is a validated instrument composed of $22 \mathrm{nu}-$ merically scaled questions rated from 0 (no fatigue) to 10 (severe fatigue) [21,22]. These items measure four dimensions of subjective fatigue: behavioral/severity (6 items); affective/meaning (5 items); sensory (5 items); and cognitive/mood (6 items). These are used to calculate the four sub-scale/dimensional scores and the total fatigue scores. The standardized alpha (Cronbach's alpha) coefficient of reliability did not drop below 0.960 for any of the subscale data, and the standard alpha for the entire scale of 22 questions was 0.979 , indicating excellent reliability and internal consistency for this established instrument [23]. The study participants took the PFS survey at days $0,7,30$ and 60 .

\subsection{Subjects}

Participants were prescreened after an initial contact to determine whether their symptoms were consistent with persistent, intractable fatigue, or merely an intermittent condition linked to their work, lifestyle or sleep patterns. Those who described a condition consistent with the definition of moderate to severe fatigue as defined in the PFS [20-22] were directed to take an online survey after they completed an Informed Consent document. A follow-up was conducted to determine any physician's diagnosis as well as complete description of their condition and laboratory tests that were used to confirm their diagnosis (Table 2). Potential subjects were also asked how long their condition existed, whether it had been affected by any drug or supplement, and how many physicians they had been to before entering the trial. They were also asked if they were currently using any prescription medications to see if this might exclude them from the study, as determined previously [26]. The completed online surveys were scored as described previously [27].

After the initial PFS survey and confirmation of their condition, 68 participants with an overall PFS fatigue score greater than 5 were admitted to this open-label pilot study, if their fatigue could not be explained by a preexisting clinical condition. There were 58 respondents (30 females and 28 males) who were fully compliant and completed the study. The mean age \pm standard deviation (SD) of participants completing the study was $55.0 \pm 1.4$ years (females, $51.8 \pm 1.7$ years and males, $58.5 \pm 2.1$ years, respectively).

\subsection{Study Design}

Subjects signed an informed consent document and were admitted into the study with measurable moderate to severe fatigue (5 - 10 in the overall average PFS). Each participant was given instructions to take the glycophospholipid supplement with NADH and CoQ10 daily after an initial PFS assessment (time 0). All subjects repeated the PFS assessment at the end of the first week on line without access to their previous scores. After the participants completed their PFS questionnaires at 30 and 60 days, all of the electronic forms were checked for verification, completion and scoring accuracy [27].

\subsection{Statistics}

Data were analyzed by ANOVA, with significance defined as $P<0.05$. Further data analysis was performed with Tukey test and linear regression analysis, with significance defined as $P<0.05$. The standardized alpha (Cronbach's alpha) was used to confirm reliability and internal consistency of the data [23].

Table 2. Study participants’ diagnosis.

\begin{tabular}{cll}
\hline No. Patients & \multicolumn{1}{c}{ Diagnoses $^{* *}$} & \multicolumn{1}{c}{ Notes on Diagnoses } \\
\hline 30 & Chronic Fatigue Syndrome & $\begin{array}{l}\text { Myalgic encephalomyelitis (CFS/ME) Canadian } \\
\text { consensus definition [24] }\end{array}$ \\
17 & Lyme Disease & Symptoms, positive Western blot analysis [25] \\
4 & Other Fatiguing Illnesses & Fibromyalgia syndrome, Gulf War illness \\
2 & Autoimmune Disease & Rheumatoid arthritis \\
& Cancer & Active, secondary disease \\
& Diabetes & Active, under control
\end{tabular}

*Some patients had more than one diagnosis; ${ }^{* *}$ Diagnosis made by physician of record. 


\section{Results}

\subsection{Subjects in the Study}

The subjects in this study were long-term chronic illness patients who had moderate to severe chronic fatigue for years without improvement (mean \pm SD years without improvement of fatigue $=17.1 \pm 0.6$ years). Most had been to multiple physicians (mean $\pm \mathrm{SD}=15.2 \pm 0.7$ ) and had taken a variety of drugs and supplements (mean \pm SD $=35.4 \pm 2.7$ ) without expected relief of their fatigue symptoms. As expected, the majority of the subjects $(\mathrm{n}=$ 30) had a diagnosis of chronic fatigue syndrome/myalgic encephalomyelitis (Canadian consensus definition), but there was a significant group $(n=17)$ that had chronic Lyme disease (over two years, confirmed by Western blot analysis) or other fatiguing illnesses $(\mathrm{n}=16$, fibromyalgia syndrome or Gulf War illness) (Table 2). Of the 68 subjects that started the study, 58 completed the study. Almost all of the subjects that withdrew from the study did so without ever completing the day 0 PFS survey and taking the test supplement, or they were omitted from the data analysis for non-compliance during the trial. One participant left the trial because of severe headaches, which had occurred intermittently before the trial, and one participant left because of gastrointestinal complaints. In both cases these symptoms resolved after stopping the test supplement, and no further complaints were recorded.

\subsection{Effects of Test Supplement on Fatigue}

We examined the effects of the combination glycophospholipid-NADH-CoQ10 preparation used in this study (Table 1) on fatigue scores in a 2-month open label trial and found significant improvements in the overall fatigue scores of the 58 study subjects as measured by the PFS (Table 3). The initial PFS group mean total fatigue score \pm SD was $7.51 \pm 0.29$, and after 8 weeks of supplement this improved to $5.21 \pm 0.28$, or a $30.7 \%$ reduction in fatigue. The mean decrease in fatigue scores was significant by t-test $(P<0.0001)$ and Wilcoxon signed-rank $(P$ $<0.0001)$ analyses.

The PFS can be further dissected into subcategories that include: behavior/severity, affective meaning, sensory and cognitive/mood (Table 3). All of these subcategories showed significant reductions at the end of the 8-week trial $(P<0.0001)$, indicating that there were significant improvements in all subcategories of fatigue. For example, there was a $30.7 \%$ reduction $(P<0.0001)$ in severity/behavior of fatigue, indicating that there was a significant reduction in the intensity of fatigue, and a significant increase in the ability to complete tasks, socialize and engaging in sexual and other activities. Also, there was a $28.0 \%$ improvement $(P<0.0001)$ in mood and cognitive ability, such as the ability to concentrate, remember and think clearly (Table 3).

\subsection{Regression Analysis of Fatigue Data}

To see if the trends in fatigue reduction over time during the trial were consistent, occurred with a high degree of confidence and could predict further reductions we conducted regression analyses of the data. As shown in Figure 1, the regression analysis of overall fatigue in each of the subcategories of fatigue (behavior/severity, affective meaning, sensory and cognitive/mood) indicated significant and consistent downward trends in the fatigue data, suggesting that further reductions in fatigue would have been likely if the trial had been continued (Figure 1). The regression $\mathrm{R}^{2}$ values for the various subgroups were: behavior/severity, 0.956; affective meaning, 0.960; sensory, 0.950; and cognitive/mood, 0.980. Regression analysis of the overall fatigue yielded a $R^{2}=0.960$. This indicates a high level of confidence in the downward trends in all fatigue data.

Table 3. Results from overall fatigue and subcategories of the piper fatigue scale survey.

\begin{tabular}{ccccc}
\hline Category & $\begin{array}{c}\text { Mean Fatigue } \\
\text { Day 0 }\end{array}$ & $\begin{array}{c}\text { Level } \pm \text { S.E.M. } \\
\text { Day 60 }\end{array}$ & $\begin{array}{c}\text { Percent } \\
\text { Reduction }\end{array}$ & $\begin{array}{c}\text { t-test } \\
P\end{array}$ \\
\hline Overall Fatigue & $7.51 \pm 0.29$ & $5.21 \pm 0.28$ & 30.7 & $<0.0001$ \\
Behavior/Severity & $7.43 \pm 0.26$ & $5.15 \pm 0.12$ & 30.7 & $<0.0001$ \\
Affective/Meaning & $8.36 \pm 0.14$ & $5.90 \pm 0.16$ & 29.4 & $<0.0001$ \\
Sensory & $7.75 \pm 0.22$ & $5.49 \pm 0.17$ & 29.2 & $<0.0001$ \\
Cognitive/Mood & $6.40 \pm 0.24$ & & & $<0.0001$ \\
\hline
\end{tabular}




\subsection{Regression Analysis of Data Based on Sex}

We examined participants' data to see if there was any difference between males and females. Differences between the response to the test supplement in males and females were obvious in the regression analyses (Figure 2). Females showed more fatigue at the beginning of the trial as well as at the end compared to males (Figure 2). Males showed an average of $35.9 \%$ improvement in overall fatigue by the end of the trial $(P<0.0001)$, whereas females ended the trial with an average improvement in fatigue of $23 \%(P<0.0001)$. The regression analysis $\mathrm{R}^{2}$ values for males $=0.951$, and for females $\mathrm{R}^{2}=0.970$. This indicates a high level of confidence in the fatigue data in both males and females.

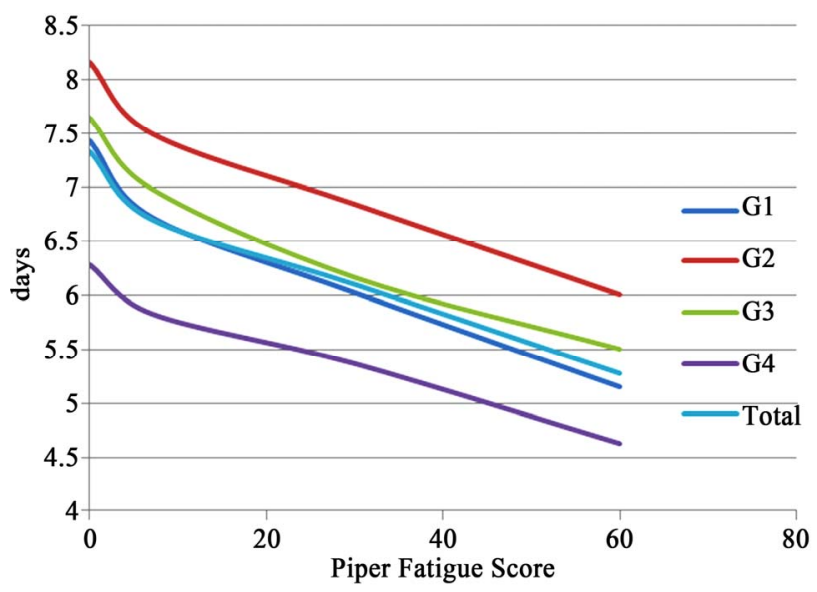

Figure 1. Data regression analyses of various patient subgroups taking the test supplement in a 60-day trial. $X$ axis, days; $Y$ axis, Piper Fatigue Score. The regression $R^{2}$ values for the various subgroups were: $\mathrm{G1}$ : behavior/severity, 0.956; G2: affective meaning, 0.960; G3: sensory, 0.950; and G4: cognitive/mood, 0.980 . Total: regression analysis of the overall fatigue, $R^{2}=0.960$.

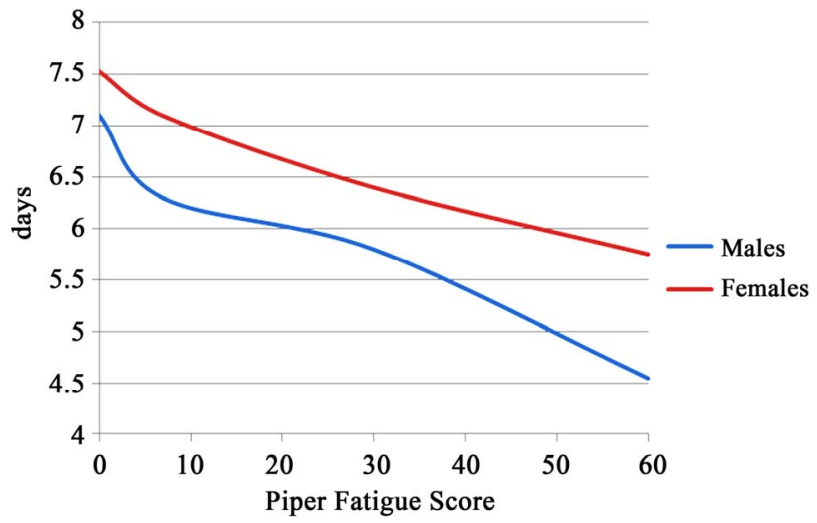

Figure 2. Data regression analyses of male and female subgroups taking the test supplement in a 60-day trial. $X$ axis, days; $Y$ axis, Piper Fatigue Score. The regression $R^{2}$ values for males was 0.961 , and females, 0.970 .

\subsection{Analysis of Data Based on Severity or Diagnosis}

We also examined the data to see if there was a difference in response to the test supplement that was dependent on fatigue severity. As expected from previously trials that used NT Factor ${ }^{\circledR}$ alone [28], the most severely fatigued subjects showed the greatest reductions in fatigue scores. For example, subjects with initial overall fatigue scores above 8 in the PFS scale showed greater reductions in fatigue scores on day 60 (35.3\% improvement in overall fatigue) than subjects with lower scores (intial PFS score 4 - 8, 25\% improvement by day 60). Examination of chronic fatigue syndrome/myalgic encephalomyelitis, Lyme disease or other diagnosis categories did not reveal major differences in overall fatigue or its reduction by the test supplement that were related to diagnosis. For example, the CFS/ME, Lyme disease and other fatiguing illness groups total fatigue was reduced on day 60 by $29.6 \%, 27 \%$ and $34.6 \%$, respectively. The differences between the diagnosis groups on day 60 were not significant. However, in these diagnosis subgroups differences between initial overall fatigue at day 0 and overall fatigue on day 60 were highly significant $(P<$ 0.0001).

\section{Discussion}

LRT using the glycophospholipid supplement NT Factor $^{\circledR}$ has been used successfully in animal and clinical lipid replacement studies [6,12,20,26-28]. In this formulation encapsulated lipids are protected from oxidation in the gut and can be absorbed and transported into tissues without oxidative damage. NT Factor ${ }^{\circledR}$ contains a variety of components, including phospholipids, glycophospholipids and other lipids, nutrients, probiotics, vitamins, minerals and plant extracts $[6,12]$.

In animal studies NT Factor ${ }^{\circledR}$ has been used to prevent hearing loss associated with aging [30]. Seidman et al. [30] found that NT Factor ${ }^{\circledR}$ prevented hearing loss associated with aging and shifted the threshold hearing from 35 - $40 \mathrm{~dB}$ in control aged animals to $13-17 \mathrm{~dB}$ in the treatment group $(P<0.005)$. They also found that NT Factor $^{\circledR}$ preserved cochlear mitochondrial function. NT Factor $^{\circledR}$ also prevented aging-related mitochondrial DNA deletions found in the cochlear [30].

LRT has also been successfully used in clinical studies to reduce fatigue and protect cellular and mitochondrial membranes from oxidative damage $[6,12]$. For example, NT Factor ${ }^{\circledR}$ has been used in a vitamin and mineral mixture (Propax ${ }^{\mathrm{TM}}$ ) in cancer patients to reduce the effects of cancer therapy, such as chemotherapy-induced fatigue, nausea, vomiting and other side effects associated with chemotherapy [30,31]. In a double-blinded, cross-over, 
placebo-controlled, randomized trial on cancer patients receiving chemotherapy Colodny et al. [30] showed that LRT improved fatigue, nausea, diarrhea, impaired taste, constipation, insomnia and other quality of life indicators.

NT Factor ${ }^{\circledR}$ has been used in a study with severe chronic fatigued patients to reduce their fatigue [26]. We found that fatigue was significantly reduced from severe to moderate fatigue, after eight weeks of LRT supplementation with NT Factor ${ }^{\circledR}$ [26]. We also examined the effects of NT Factor ${ }^{\circledR}$ on fatigue in moderately and mildly fatigued subjects and determined if their mitochondrial function improved with administration of NT Factor $^{\circledR}$ [28]. Use of NT Factor ${ }^{\circledR}$ for 12 weeks resulted in 35.5\% reduction in fatigue $(P<0.001)$. In this clinical trial there was good correspondence between reductions in fatigue and gains in mitochondrial function. After 8 weeks of LRT with NT Factor ${ }^{\circledR}$, mitochondrial function was significantly improved $(P<0.001)$, and after 12 weeks of NT Factor ${ }^{\circledR}$ supplementation, mitochondrial function was found to be similar to that of young healthy adults [28]. After 12 weeks of supplement use, subjects were placed on placebo without their knowledge for an additional 12 weeks, and their fatigue and mitochondrial function were again measured. After the 12-week placebo period fatigue and mitochondrial function were intermediate between the initial starting values and those found after eight or 12 weeks on supplement, indicating that continued supplementation is likely required to show improvements in mitochondrial function and to maintain lower fatigue scores [28]. Similar results were found with chronic fatigue syndrome and/or fibromyalgia syndrome patients [20]. Thus the results were similar to those found here for CFS/ME, Lyme disease, fibromyalgia syndrome and Gulf War illness patients.

Here we used a supplement preparation containing NT Factor $^{\circledR}$, CoQ10 and microencapsulated NADH. Prior studies reported that microencapsulated NADH alone had a positive effect on fatigue in some patients. Analysis of those studies indicated that a subset of patients responded or the response was for a limited time. In the randomized, cross-over study of Forsyth et al. [32] on CFS/ME patients 8 of 26 (30.7\%) responded to microencapsulated NADH compared to 2 of $26(8 \%)$ on placebo $(P<0.05)$. However, these results were not considered significant by others [33,34]. When Santaella et al. [35] compared the effectiveness of oral NADH versus a combination of psychological/nutritional therapy for $31 \mathrm{CFS} / \mathrm{ME}$ patients during a 24-month clinical trial, they found that NADH demonstrated a reduction in fatigue in the first trimester of the trial. In subsequent trimesters, symptom scores were similar in NADH and psychological/nutritional therapy arms of the trial. In another study oral NADH was given for two months to CFS/ME patients. While the NADH group showed a decrease in anxiety and maximum heart rate after a stress test, differences were not found in the functional impact of fatigue, quality of life, sleep quality, exercise capacity or functional reserve between the test and placebo group [36].

$\mathrm{NADH}$ is an important cofactor that is required in many cellular enzyme pathways, including those involving certain transferases, polymerases, synthases, deacetylases and other enzymes [37]. It is also important in signal transduction, DNA repair, salvage pathways and posttranslational protein modifications [38]. NADH in its reduced form is a product of the mitochondrial electron transport chain, and it plays an important role in programmed cell death [39]. Thus the NAD+/NADH system has a major physiological impact on cellular health and metabolism [40]. When mitochondria are functioning properly, enough NADH is produced to meet cellular requirements [39].

CoQ10 is an essential electron carrier in the mitochondrial respiratory chain and an important antioxidant as well as a molecule involved in gene regulation [41,42]. It has been used as an important supplement in a variety of chronic illnesses and age-related conditions [42-44]. Here CoQ10 was used to improve energy transduction and combat oxidative stress $[42,45]$. This cofactor has been used extensively for combined nutraceutical therapy in mitochondrial cytopathies $[45,46]$.

The present study used a combination nutraceutical formulation containing NT Factor ${ }^{\circledR}$, CoQ10 and NADH and included a variety of chronic illness patients who had been sick with chronic fatigue for an average of over 17 years. Chronic fatigue is known to cause loss of mitochondrial function [28,46]. These patients had tried a large number of supplements and drugs (over 35) with little or no effect on their fatigue. Thus this sample of patients could be considered as having intractable fatigue, yet they responded quite well to the combination supplement containing NT Factor ${ }^{\circledR}$, CoQ10 and NADH. In addition, regression analysis indicated that the data were consistent with a high degree of confidence. The regression analysis also suggested that the trial may have ended too soon, and the peak benefits on fatigue were yet to be realized. The supplement was safe, and only two participants experienced minor symptoms that resolved after the supplement was discontinued and could not be directly attributed to the effects of the test supplement since their problems preceded entry into the trial. Thus the clinical trial demonstrated that the test supplement was effective and safe for long-term chronic illness patients.

\section{Conclusion}

After 60 days use of a supplement containing a mixture of phosphoglycolipids, CoQ10 and NADH, fatigue meas- 
ured with the validated Piper Fatigue Scale was significantly reduced in 58 patients with chronic fatigue syndrome/myalgic encephalomyelitis, chronic Lyme disease or other fatiguing illnesses, such as fibromyalgia syndrome or Gulf War illness. The supplement was safe and effective and reduced overall fatigue $30.8 \%$ in these longterm patients with intractable chronic fatigue.

\section{Acknowledgements}

The authors would like to thank Researched Nutritionals, Inc. and the Institute for Molecular Medicine for clinical trial financial support.

\section{REFERENCES}

[1] D. Chambers, A.-M. Bagnall, S. Hempel and C. Forbes, "Interventions for the Treatment, Management and Rehabilitation of Patients with Chronic Fatigue Syndrome/ Myalgic Encepthalomyelitis: An Updated Systematic Review," Journal of the Royal Society of Medicine, Vol. 99, No. 10, 2006, pp. 506-520. doi:10.1258/jrsm.99.10.506

[2] S. Reid, T. Chalder, A. Cleare, M. Hotopf and S. Wessley, “Chronic Fatigue Syndrome,” Clinical Evidence, Vol. 2011, 2011, p. 1101.

[3] J. D. Morrison, "Fatigue as a Presenting Complaint in Family Practice,” Journal of Family Practice, Vol. 10, No. 5, 1980, pp. 795-801.

[4] K. Kroenke, D. R. Wood, A. D. Mangelsdorff, N. J. Meier and J. B. Powell, "Chronic Fatigue in Primary Care: Prevalence, Patient Characteristics, and Outcome,” Journal of the American Medical Association, Vol. 260, No. 7, 1988, pp. 929-934.

doi:10.1001/jama.1988.03410070057028

[5] D. Purvis, S. Gonsalves and P. A. Deuster, "Physiological and Psychological Fatigue in Extreme Conditions: Overtraining and Elite Athletes," Physical and Medical Rehabilitation, Vol. 2, No. 5, 2010, pp. 442-450.

[6] G. L. Nicolson and R. Settineri, "Lipid Replacement Therapy: A Functional Food Approach with New Formulations for Reducing Cellular Oxidative Damage, CancerAssociated Fatigue and the Adverse Effects of Cancer Therapy," Functional Foods in Health and Disease, Vol. 1, No. 4, 2011, pp. 135-160.

[7] A. J. Knicker, I. Renshaw, A. R. Oldham and S. P. Cairns, "Interactive Processes Link the Multiple Symptoms of Fatigue in Sports Competition,” Sports Medicine, Vol. 41, No. 4, 2011, pp. 307-328. doi:10.2165/11586070-000000000-00000

[8] M. D. Brand and D. G. Nicholis, "Assessing Mitochondrial Dysfunction in Cells,” Biochemical Journal, Vol. 435, No. Pt2, 2011, pp. 297-312. doi:10.1042/BJ20110162

[9] M. R. Duchen and G. Szabadkai, "Roles of Mitochondria in Human Disease,” Essays in Biochemistry, Vol. 47, 2010, pp. 115-137. doi:10.1042/bse0470115

[10] B. Halliwell, “Oxidative Stress, Nutrition and Health,” Free
Radical Research, Vol. 25, No. 1, 1996, pp. 57-74.

[11] J. Montero, M. Mari, A. Colell, A. Morales, G. Basanez, C. Garcia-Ruiz and J. C. Fernandez-Checa, "Cholesterol and Peroxidized Cardiolipin in Mitochondrial Membrane Properties, Permeabilization and Death,” Biochimica Biophysica Acta, Vol. 1797, No. 6-7, 2010, pp. 1217-1224.

[12] G. L. Nicolson, "Lipid Replacement as an Adjunct to Therapy for Chronic Fatigue, Anti-Aging and Restoration of Mitochondrial Function,” Journal of the American Nutraceutical Association, Vol. 6, No. 3, 2003, pp. 22-28.

[13] R. M. Adibhatla and J. F. Hatcher, "Lipid Oxidation and Peroxidation in CNS Health and Disease: From Molecular Mechanisms to Therapeutic Opportunities," Antioxidant and Redox Signaling, Vol. 12, No. 1, 2010, pp. 125-169. doi:10.1089/ars.2009.2668

[14] T. Kanno, E. E. Sato, S. Muranaka, H. Fujita, T. Fujiwara, T. Utsumi, M. Inoue and K. Utsumi, "Oxidative Stress Underlies the Mechanism for $\mathrm{Ca}(2+)$-Induced Permeability Transition of Mitochondria," Free Radical Research, Vol. 38, No. 1, 2004, pp. 27-35. doi:10.1080/10715760310001626266

[15] E. Stadtman, "Introduction to Serial Reviews on Oxidatively Modified Proteins in Aging and Disease," Free Radical Biology and Medicine, Vol. 32, No. 9, 2002, p. 789. doi:10.1016/S0891-5849(02)00764-5

[16] L. Castro and B. A. Freeman, "Reactive Oxygen Species in Human Health and Disease," Nutrition, Vol. 17, No. 2 , 2001, pp. 295-307. doi:10.1016/S0899-9007(00)00570-0

[17] J. H. Huang and D. A. Hood, “Age-Associated Mitochondrial Dysfunction in Skeletal Muscle: Contributing Factors and Suggestions for Long-Term Interventions,” IUBMB Life, Vol. 61, No. 3, 2009, pp. 201-214. doi:10.1002/iub.164

[18] K. Green, M. D. Brand and M. P. Murphy, "Prevention of Mitochondrial Oxidative Damage as a Therapeutic Strategy in Diabetes,” Diabetes, Vol. 54, Suppl. 1, 2004, pp. S110-S118.

[19] K. Jomova, D. Vondrakova, M. Lawson and M. Valko, "Metals, Oxidative Stress and Neurodegenerative Disorders," Molecular and Cellular Biochemistry, Vol. 345, No. 1-2, 2010, pp. 91-104. doi:10.1007/s11010-010-0563-X

[20] G. L. Nicolson and R. R. Ellithrope, "Lipid Replacement and Antioxidant Nutritional Therapy for Restoring Mitochondrial Function and Reducing Fatigue in Chronic Fatigue Syndrome and Other Fatiguing Illnesses," Journal of Chronic Fatigue Syndrome, Vol. 13, No. 1, 2006, pp. 57-68. doi:10.1300/J092v13n01 06

[21] B. F. Piper, A. M. Linsey and M. J. Dodd, "Fatigue Mechanism in Cancer," Oncology Nursing Forum, Vol. 14, 1987, pp. 17-23.

[22] B. F. Piper, S. L. Dribble and M. J. Dodd, “The Revised Piper Fatigue Scale: Psychometric Evaluation in Women with Breast Cancer,” Oncology Nursing Forum, Vol. 25, 1998, pp. 667-684.

[23] J. M. Bland and D. G. Altman, "Statistical Notes: Cronbach’s Alpha,” British Medical Journal, Vol. 314, No. 7080, 1997, p. 572. doi:10.1136/bmj.314.7080.572

[24] B. M. Crothers, A. K. Jain, K. L. De Meirleir, D. L. Pe- 
terson, N. G. Klimas, A. M. Lerner, A. C. Bested, P. FlorHenry, P. Joshi, A. C. P. Powles, J. A. Sherkey and M. I. van de Sande, "Myalgic Encephalomyelitis/Chronic Fatigue Syndrome: Clinical Working Case Definition, Diagnostic and Treatment Protocols," Journal of Chronic Fatigue Syndrome, Vol. 11, No. 1, 2003, pp. 7-116.

[25] J. J. Burrascano Jr., "Advanced Topics in Lyme Disease: Diagnostic Hints and Treatment Guidelines for Lyme and Other Tick-Borne Illnesses,” 16th Edition, International Lyme and Associated Diseases Society, Bethesda, 2008, pp. 1-37.

[26] R. R. Ellithorpe, R. Settineri and G. L. Nicolson, "Reduction of Fatigue by Use of a Dietary Supplement Containing Glycophospholipids," Journal of the American Nutraceutical Association, Vol. 6, No. 1, 2006, pp. 23-28.

[27] G. L. Nicolson, R. R. Ellithorpe, C. Ayson-Mitchell, B. Jacques B and R. Settineri, "Lipid Replacement Therapy with a Glycophospholipid-antioxidant-vitamin Formulation Significantly Reduces Fatigue within One Week,” Journal of the American Nutraceutical Association, Vol. 13, No. 1, 2010, pp. 11-15.

[28] M. Agadjanyan, V. Vasilevko, A. Ghochikyan, P. Berns, P. Kesslak, R. Settineri and G. L. Nicolson, "Nutritional Supplement (NTFactor) Restores Mitochondrial Function and Reduces Moderately Severe Fatigue in Aged Subjects,” Journal of Chronic Fatigue Syndrome, Vol. 11, No. 3, 2003, pp. 23-36.

[29] M. Seidman, M. J. Khan, W. X. Tang and W. S. Quirk, "Influence of Lecithin on Mitochondrial DNA and AgeRelated Hearing Loss,” Otolaryngology and Head and Neck Surgery, Vol. 127, No. 3, 2002, pp. 138-144. doi:10.1067/mhn.2002.127627

[30] L. Colodny, K. Lynch, C. Farber, S. Papish, K. Phillips, M. Sanchez, K. Cooper, O. Pickus, D. Palmer, T. B. Percy, M. Faroqui and J. B. Block, "Results of a Study to Evaluate the Use of Propax to Reduce Adverse Effects of Chemotherapy," Journal of the American Nutraceutical Association, Vol. 3, No. 1, 2000, pp. 17-25.

[31] G. L. Nicolson, "Lipid Replacement Therapy: A Nutraceutical Approach for Reducing Cancer-Associated Fatigue and the Adverse Effects of Cancer Therapy while Restoring Mitochondrial Function," Cancer and Metastasis Reviews, Vol. 29, No. 3, 2010, pp. 543-552. doi:10.1007/s10555-010-9245-0

[32] L. M. Forsyth, H. G. Preuss, A. L. MacDowell, L. Chiazze Jr., G. D. Birkmayer and J. A. Bellanti, “Therapeutic Effects of Oral NADH on the Symptoms of Patients with Chronic Fatigue Syndrome," Annals of Allergy, Asthma and Immunology, Vol. 82, No. 2, 1999, pp. 185-191. doi:10.1016/S1081-1206(10)62595-1

[33] D. Colquhoun and S. Senn, "Is NADH Effective in the Treatment of Chronic Fatigue Syndrome?” Annals of Allergy, Asthma and Immunology, Vol. 84, No. 6, 2000, pp. 639-640. doi:10.1016/S1081-1206(10)62421-0

[34] D. Chambers, A. M. Bagnall, S. Hempel and C. Forbes, "Interventions for the Treatment, Management and Rehabilitation of Patients with Chronic Fatigue Syndrome/ Myalgic Encephalomyelitis: An Updated Systematic Review," Journal of the Royal Society of Medicine, Vol. 99,
No. 10, 2006, pp. 506-520. doi:10.1258/jrsm.99.10.506

[35] M. L. Santaella, I. Font and O. M. Disdier, "Comparison of Oral Nicotinamide Adenine Dinucleotide (NADH) versus Conventional Therapy for Chronic Fatigue Syndrome," Puerto Rico Health Science Journal, Vol. 23, No. 2, 2004, pp. 89-93.

[36] J. Alegre, J. M. Rosés, C. Javierre, A. Ruiz-Baqués, M. J. Segundo and T. F. de Sevilla, "Nicotinamide Adenine Dinucleotide (NADH) in Patients with Chronic Fatigue Syndrome,” Reviews Clinica España, Vol. 210, No. 6, 2010, pp. 284-288. doi:10.1016/j.rce.2009.09.015

[37] P. Belenky, K. L. Bogan and C. Brenner, "NAD+ Metabolism in Health and Disease," Trends in Biochemical Science, Vol. 32, No. 1, 2007, pp. 12-19. doi:10.1016/j.tibs.2006.11.006

[38] L. Chen, R. Petrelli, K. Felczak, G. Gao, L. Bonnac, Y. S. Yu, E. M. Bennett and K. W. Pankiewicz, "Nicotinamide Adenine Dinucleotide Based Therapeutics," Current Medicinal Chemistry, Vol. 15, No. 7, 2008, pp. 650-670. doi:10.2174/092986708783885282

[39] W. Ying, "NAD+ and NADH in Cellular Functions and Death,” Frontiers in Bioscience, Vol. 11, 2006, pp. 31293148. doi:10.2741/2038

[40] N. Kashihara, Y. Haruna, V. K. Kondeti and Y. S. Kanwar, "Oxidative Stress in Diabetic Nephropathy," Current Medicinal Chemistry, Vol. 17, No. 34, 2010, pp. 42564269. doi:10.2174/092986710793348581

[41] C. M. Quinzii and M. Hirano, "Coenzyme Q and Mitochondrial Disease,” Developmental Disabilities Research Reviews, Vol. 16, No. 2, 2010, pp. 183-188. doi:10.1002/ddrr.108

[42] G. P. Littarru and L. Tiano, "Clinical Aspects of Coenzyme Q10: An Update,” Nutrition, Vol. 26, No. 3, 2010, pp. 250254. doi:10.1016/j.nut.2009.08.008

[43] D. Orsucci, M. Filosto, G. Siciliano and M. Mancuso, "Electron Transfer Mediators and Other Metabolites and Cofactors in the Treatment of Mitochondrial Dysfunction," Nutrition Reviews, Vol. 67, No. 9, 2009, pp. 427438. doi:10.1111/j.1753-4887.2009.00221.x

[44] M. Mancuso, D. Orsucci, L. Volpi, V. Calsolaro and G. Siciliano, "Coenzyme Q10 in Neuromuscular and Neurodegenerative Disorders,” Current Drug Targets, Vol. 11, No. 1, 2010, pp. 111-121. doi:10.2174/138945010790031018

[45] M. A. Tarnopolsky, "The Mitochondrial Cocktail: Rationale for Combined Nutraceutical Therapy in Mitochondrial Cytopathies," Advances in Drug Delivery Reviews, Vol. 60, No. 13-14, 2008, pp. 1561-1567. doi:10.1016/j.addr.2008.05.001

[46] S. Beg, S. Javed and K. Kohli, "Bioavailability Enhacement of Coenzyme Q10: An Extensive Review of Patents," Recent Patents on Drug Delivery and Formulation, Vol. 4, No. 3, 2010, pp. 245-255. doi:10.2174/187221110793237565

[47] S. Myhill, N. E. Booth and J. McLaren-Howard, "Chronic Fatigue Syndrome and Mitochondrial Dysfunction,” International Journal of Clinical and Experimental Medicine, Vol. 2, No. 1, 2009, pp. 1-16. 